INFLAMMATORY BOWEL DISEASE

\title{
Appendicectomy has no beneficial effect on admission rates in patients with ulcerative colitis
}

\author{
J Hallas, D Gaist, W Vach, H T Sørensen
}

Gut 2004;53:351-354. doi: 10.1136/gut.2003.016915

See end of article for authors' affiliations

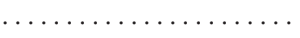

Correspondence to: Dr J Hallas, Department of Internal Medicine, Odense University Hospital, 5000 Odense C, Denmark; i.hallas@dadlnet.dk

Accepted for publication 15 October 2003
Background and aims: Those who have had an appendicectomy have a reduced risk of developing ulcerative colitis. However, the effect of appendicectomy on disease activity in patients with ulcerative colitis has not been established.

Methods: We used the Danish National Patient Registry to identify all incident cases of ulcerative colitis in Denmark during the period 1981 to 1999. Of these, 202 had an appendicectomy after their first admission with ulcerative colitis. In these patients, we compared the incidence rate of hospitalisations with ulcerative colitis as first diagnosis during the period between the onset of ulcerative colitis and appendicectomy, with the rate of such hospitalisations after appendicectomy. To adjust for the clinical course of ulcerative colitis unrelated to appendicectomy, we extracted a reference cohort $(n=808)$, matched to the index subjects with respect to age, sex, and year of first admission, but with an intact appendix.

Results: The rate of admission with ulcerative colitis as first diagnosis decreased by $47 \%$ after appendicectomy (rate ratio 0.53 (95\% confidence interval (CI) 0.36-0.80)). However, the reference cohort showed a similar decline in admission rate (rate ratio 0.51 ). Thus appendicectomy had no apparent beneficial effect on admission rate after adjustment for the clinical course of disease unrelated to appendicectomy (adjusted rate ratio 1.05 (95\% Cl 0.67-1.67)).

Conclusions: Appendicectomy had no significant beneficial effect on admission rates in patients with ulcerative colitis.
A number of studies have shown that patients who have had an appendicectomy have a lower incidence of ulcerative colitis (UC) than those with an intact appendix. ${ }^{1-7}$ Thus appendicectomy seems to be inversely related to the risk of UC. However, the prognostic impact on the clinical course of UC is unknown.

From a clinical viewpoint, the obvious question is whether this might have some clinical use, for example prophylactic appendicectomy in subjects at risk of developing UC or therapeutic appendicectomy to ameliorate the course of disease in patients who already have UC. With regard to the use of appendicectomy as a prophylactic measure, it would be difficult to identify subjects with a risk of UC that by itself would justify an appendicectomy; even first degree relatives of UC patients have only a $1-5 \%$ prevalence of UC. ${ }^{8-10}$ If, on the other hand, appendicectomy could lower the risk of relapse in patients with established UC, it would have tremendous clinical utility. This question has not been adequately addressed in previous publications on the UCappendicectomy association. To evaluate the potential of therapeutic appendicectomy, we conducted a study of disease activity in patients who had an appendicectomy after the onset of UC.

\section{MATERIALS AND METHODS}

\section{Danish National Registry of Patients (DNRP)}

Since 1977, data on all individual discharges from public hospitals in Denmark have been stored in the Danish National Registry of Patients (DNRP). ${ }^{11}$ Each record contains a personal registration number (a unique identifier of all Danish citizens), as well as selected medical data, including admission dates, discharge diagnoses, and operative procedures. Virtually all inpatient medical care in Denmark is provided by the public health authorities, thus allowing true population based studies of Denmark's 5.2 million inhabi- tants. The discharge coding was based on ICD-8 for the period 1977-1993 and on ICD10 after 1993. ICD9 has never been used in Denmark. Coding is performed by physicians on the basis of discharge summaries.

\section{The study population}

As part of another study on the appendicectomy-UC association, we used the DNRP to identify all 234559 subjects who had an appendicectomy in Denmark during the period January 1977 to December 1999.

To obtain data on incident cases of UC, all patients discharged with a diagnosis of UC or haemorrhagic proctitis during the period January 1977 to December 1999 were retrieved from the DNRP and considered for inclusion in the study $(\mathrm{n}=21 \mathrm{769})$. Since we were only interested in studying incident cases, we subsequently excluded those who had their first admission with a recorded UC diagnosis during the years 1977-1980. These were likely to be a mixture of incident and non-incident cases in whom UC onset could not be validly determined $(\mathrm{n}=5482)$. We also excluded those who ever had a modifier code of "suspected" or "not confirmed" UC diagnosis $(\mathrm{n}=3191)$, or ever had a diagnosis of Crohn's disease (ICD-8: 563.00-563.09, ICD-10; K50) $(\mathrm{n}=1166)$. The remaining 11930 patients comprised 5844 men and 6086 women. For members of this cohort, we defined UC onset as the date of the first hospitalisation with a diagnosis of UC.

For the purpose of censoring, we also retrieved data on the date of death, emigration, or immigration of subjects from the Danish Central Person Registry. In addition, we retrieved

Abbreviations: UC, ulcerative colitis; DNRP, Danish National Registry of Patients 
data on colectomies from the DNRP. Data on discharge diagnoses, surgical procedures, migrations, and deaths were linked by use of the personal identification numbers.

\section{Index cohort}

By merging the cohorts of UC patients and those who had an appendicectomy, we identified 202 subjects who had an appendicectomy after their onset of UC. These were termed the index cohort

We used the incidence rate of hospital admissions with UC as the primary diagnosis as a proxy for activity, and termed it hospital admission rate. The hospital admission rate for UC was calculated for the period before and after appendicectomy by conventional techniques. ${ }^{12}$ We counted only person time after the first admission for UC.

Follow up continued after each admission for UC until the subject was censored-that is, one of the following occurred: death, colectomy, emigration, or the end of the study period (31 December 1999), whichever event came first. The first admission with UC was disregarded in the calculation of incidences since this event defined the subject as having UC before appendicectomy and-if included-would bias the result towards a larger decline in admission rate after appendicectomy.

\section{Reference cohort}

A decline in admission rates after appendicectomy could be explained as a clinical course of UC disease-that is, that admission rates by themselves would fall over time, independent of appendicectomy. To account for this, we retrieved a reference cohort from the 11371 UC patients who had not had an appendicectomy. To optimise statistical power, we selected four matched reference subjects for each subject in the index cohort.

Each reference subject $(n=808)$ was randomly selected from those that matched the index subject with regard to sex, birth year (within five years), and first diagnosis of UC (within two years) but who had not had an appendicectomy. Each reference subject was assigned an index date equivalent to the appendicectomy date of the index subject-that is, the time interval, measured in days, between UC onset and the index date of each reference subject was equal to the interval between UC onset and appendicectomy of the corresponding index subject. The observation period from study entry to appendicectomy in the index cohort comprised immortal person time-that is, person time where the subject by definition cannot be censored. ${ }^{13}$ In order for the reference cohort to have equal conditions of observation, we required that each eligible reference subject had a follow up free of censoring at least as long as to cover the index date. Censoring occurred with the same events as for the index cohort. The design of this matching procedure is illustrated in fig 1 .

For the reference cohort, we calculated the incidence of admissions before/after the index dates by the same method as for the index cohort. The main result of our study (that is, the effect of appendicectomy on the course of UC, adjusted for clinical course unrelated to appendicectomy) was expressed as a ratio of two ratios: (1) the ratio of admission rates after/before appendicectomy for the index cohort; and (2) the ratio of admission rates after/before the index date for the reference cohort.

In computing confidence intervals $(95 \% \mathrm{CI}$ ) for the rate ratios and the ratio between the two rate ratios, we had to take into account that counts of pre- and post-events within an individual are correlated. This was accomplished using Stata's cluster option and expressing incidence rate ratios as estimates in a Poisson regression model. ${ }^{14}$

To confirm the main result, we performed a supplementary analysis confined to the index cohort in which we explored whether the admission rate would be different for person time before and after appendicectomy after adjustment for duration of disease. We stratified the follow up into different time bands according to the number of years passed since the diagnosis of UC. Within each time band, person time was categorised as either before or after appendicectomy, and the incidence rate of admissions was calculated accordingly.

\section{RESULTS}

There were 202 subjects who had an appendicectomy after their first admission for UC (table 1). Of these, 84 were men. Mean age at UC onset was 38.6 years. They contributed 953 person years of follow up before their appendicectomy and 1221 person years after. Discounting the first admission, there were 171 admissions with UC as first diagnosis before appendicectomy and 117 admission after. The incidence rates

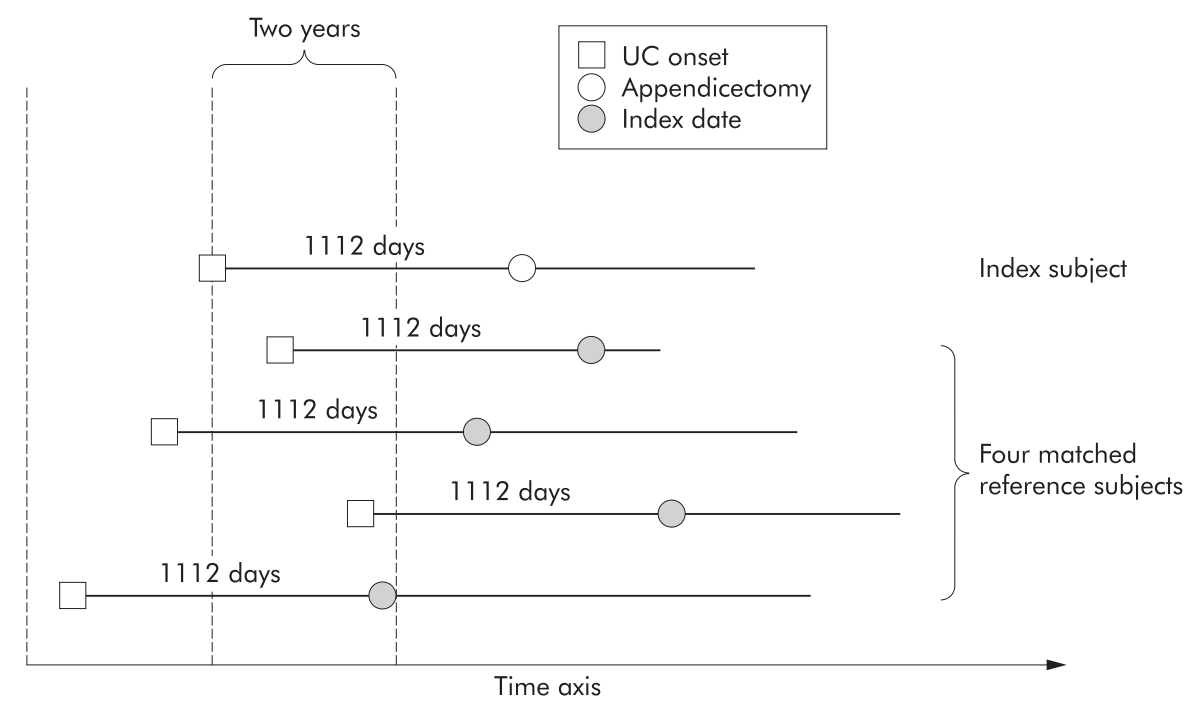

Figure 1 Design of the study. Four reference subjects were retrieved for each index subject with an appendicectomy after the onset of ulcerative colitis. Reference subjects are ulcerative colitis patients, matched with respect to sex, age (within five years), and time of onset of ulcerative colitis (within two years) but who did not have an appendicectomy. They were assigned an index date equidistant to the appendicectomy date of the index subject and followed up for a variable amount of time thereafter. 
Table 1 Characteristics of the 202 patients who had an appendicectomy after their first admission for ulcerative colitis or haemorrhagic proctitis (UC), and 808 reference subjects matched for age, sex, and time of onset. Subjects were identified in the Danish National Patient Registry, January 1981 to December 1999

\begin{tabular}{lcc}
\hline & $\begin{array}{l}\text { Patients with } \\
\text { appendicectomy } \\
(\mathbf{n = 2 0 2 )}\end{array}$ & $\begin{array}{l}\text { Reference subjects } \\
\text { (n= 808) }\end{array}$ \\
\hline Men (n) & $84(42 \%)$ & $336(42 \%)$ \\
Age at UC onset (y) & $38.6(18.0)$ & $38.7(17.7)$ \\
Age at appendicectomy/index date (y) $)^{*}$ & $43.3(17.8)$ & $43.4(17.6)$ \\
Follow up after UC onset (y) & $10.77(5.2)$ & $10.99(5.1)$ \\
Before appendicectomy/index date & $4.72(4.3)$ & $4.72(4.3)$ \\
After appendicectomy/index date & $6.05(4.7)$ & $6.26(4.7)$ \\
No of UC admissions & 288 & 1055 \\
Before appendicectomy/index date & $171(59 \%)$ & $631(60 \%)$ \\
After appendicectomy/index date & $117(41 \%)$ & $424(40 \%)$ \\
Incidence of UC admissions (per year) & & 0.1654 \\
Before appendicectomy/index date & 0.1793 & 0.0838 \\
After appendicectomy/index date & 0.0958 & \\
\hline *Values are mean (SD). & &
\end{tabular}

of UC admissions were 0.18 per year before appendicectomy and 0.10 per year after. The ratio of UC admission rates after and before appendicectomy was 0.53 (95\% CI $0.36-0.80$ ), corresponding to a $47 \%$ decrease in admission rate after appendicectomy. Of the 202 reference subjects, nine eventually had a colectomy $(4.5 \%)$.

The reference cohort consisted of 808 subjects (336 men and 472 women) with a mean age at UC onset of 38.7 years. They contributed 3815 and 5061 years of follow up before and after their index dates (table 1). Of the 808 reference subjects, 42 eventually had a colectomy $(5.2 \%)$. The incidence rate of admissions before and after the index date were 0.17 and 0.08 per year, corresponding to a post/pre ratio of 0.51 . If these values are used as a reference for the clinical course of UC unrelated to appendicectomy, we find an adjusted ratio of UC admissions after/before appendicectomy of 1.05 (95\% CI 0.67-1.67), corresponding to a 5\% higher than expected admission rate after appendicectomy.

We repeated the entire analysis specifically for the 137 subjects who had an appendicitis diagnosis and for 65 subjects without such a diagnosis at the time of appendicectomy. In each analysis we used the same main design with a 4:1 allocation of reference subjects and with assignment of index days for the reference subjects. Results are shown in table 2. There was a trend towards a suggested beneficial effect of appendicectomy in subjects without appendicitis (0.76 (95\% CI 0.51-1.19)). However, the two confidence intervals for the subgroups overlapped, and both encompassed unity.

The results of the confirmatory analysis are shown in table 3. As expected, the incidence rates decreased with increasing time elapsed since UC diagnosis but with no clear tendency towards differing incidence rates in person time before or after appendicectomy. A summary estimate of the strata specific estimates into a pooled rate ratio yielded a value of 1.02 (95\% CI $0.78-1.35$ ), suggesting no effect of appendicectomy.

\section{DISCUSSION}

In this population based nationwide study with complete follow up, we found a moderate decline in hospital admission rates for UC after appendicectomy in those who already had UC before their appendicectomy. However, this decline cannot be attributed to appendicectomy as the reference cohort showed an even greater decline in admission rate. This decline in admission rate with time is well known, ${ }^{15}$ and our design took this into account by use of a reference cohort.

A recent Australian study addressed the same issue in a case control study of 259 prevalent UC patients. The authors found no apparent effect of appendicectomy on measures of disease activity, such as disease extent, need for immunosuppressive therapy with azathioprine or 6-mercaptopurine, or colectomy. However, only eight patients were recruited who had their appendices removed after the onset of UC, and hence clinically important effects may have been overlooked. ${ }^{16}$

One of the problems in our study was that the admission rate was a crude measure of disease activity. Most UC patients have a course that rarely warrants admission ${ }^{17}$ and other factors, such as quality and access of outpatient care, may be important determinants of admission, except in severe episodes. It is thus possible that appendicectomy could decrease the number of mild flare ups without being evident in our material. It would have been attractive to conduct our study with more detailed account of the clinical course- that

Table 2 Stratification of the study sample according to whether the index subjects had appendicitis at the time of appendicectomy

\begin{tabular}{|c|c|c|c|c|c|}
\hline $\begin{array}{l}\text { Appendicitis } \\
\text { diagnosis }\end{array}$ & Cohort & $\mathrm{n}$ & $\begin{array}{l}\text { Follow up before/ } \\
\text { after index date } \\
\text { (person years) }\end{array}$ & $\begin{array}{l}\text { No of admission } \\
\text { before/after index } \\
\text { date }\end{array}$ & $\begin{array}{l}\text { Adjusted ratio of UC } \\
\text { admission rate }(95 \% \mathrm{Cl})\end{array}$ \\
\hline Present & $\begin{array}{l}\text { Index } \\
\text { Reference }\end{array}$ & $\begin{array}{l}137 \\
548\end{array}$ & $\begin{array}{c}646 / 823 \\
2586 / 3392\end{array}$ & $\begin{array}{l}108 / 84 \\
411 / 265\end{array}$ & $1.24(0.71-2.19)$ \\
\hline Absent & $\begin{array}{l}\text { Index } \\
\text { Reference }\end{array}$ & $\begin{array}{r}65 \\
260\end{array}$ & $\begin{array}{c}307 / 399 \\
1230 / 1670\end{array}$ & $\begin{array}{c}63 / 33 \\
220 / 159\end{array}$ & $0.76(0.33-1.76)$ \\
\hline All & $\begin{array}{l}\text { Index } \\
\text { Reference }\end{array}$ & $\begin{array}{l}202 \\
808\end{array}$ & $\begin{array}{r}953 / 1221 \\
3815 / 5061\end{array}$ & $\begin{array}{l}171 / 117 \\
631 / 424\end{array}$ & $1.05(0.67-1.67)$ \\
\hline
\end{tabular}

An adjusted ratio of ulcerative colitis (UC) admission rate below 1.0 suggests a beneficial effect of appendicectomy on the clinical course of UC.

$95 \% \mathrm{Cl}, 95 \%$ confidence interval. 
Table 3 Comparison of admission rates before and after appendicectomy, stratified by time since onset of ulcerative colitis (UC). The analysis is based only on subjects from the index cohort

\begin{tabular}{|c|c|c|c|c|c|c|c|c|}
\hline \multirow[b]{2}{*}{$\begin{array}{l}\text { Time since UC } \\
\text { onset (y) }\end{array}$} & \multicolumn{3}{|c|}{ Before appendicectomy } & \multicolumn{3}{|c|}{ After appendicectomy } & \multicolumn{2}{|c|}{ After/before appendicectomy } \\
\hline & $\begin{array}{l}\text { No of UC } \\
\text { admissions }\end{array}$ & $\begin{array}{l}\text { Follow up } \\
\text { (person years) }\end{array}$ & $\begin{array}{l}\text { Incidence } \\
\text { rate }\end{array}$ & $\begin{array}{l}\text { No of UC } \\
\text { admissions }\end{array}$ & $\begin{array}{l}\text { Follow up } \\
\text { (person years) }\end{array}$ & $\begin{array}{l}\text { Incidence } \\
\text { rate }\end{array}$ & $\begin{array}{l}\text { Rate } \\
\text { ratios }\end{array}$ & $\begin{array}{l}\text { Confidence } \\
\text { intervals }\end{array}$ \\
\hline $0-2$ & 83 & 177 & 0.47 & 7 & 24 & 0.3 & 0.62 & $0.24-1.34$ \\
\hline $2-4$ & 19 & 145 & 0.13 & 8 & 54 & 0.15 & 1.13 & $0.43-2.70$ \\
\hline $4-6$ & 33 & 216 & 0.15 & 31 & 160 & 0.19 & 1.26 & $0.75-2.14$ \\
\hline $6-8$ & 22 & 203 & 0.11 & 34 & 273 & 0.12 & 1.14 & $0.65-2.06$ \\
\hline $8-10$ & 10 & 125 & 0.08 & 17 & 262 & 0.06 & 0.82 & $0.35-2.00$ \\
\hline$\geqslant 10$ & 4 & 87 & 0.05 & 20 & 450 & 0.04 & 0.97 & $0.32-3.90$ \\
\hline All person time & 171 & 953 & NA & 117 & 1221 & NA & 1.02 & $0.78-1.35$ \\
\hline
\end{tabular}

is, with a prospective registering of data. Unfortunately, we cannot identify the subjects in our index cohort before they have an appendicectomy. Thus it would be impossible to gather prospective information about the clinical course before appendicectomy. In addition, the crudeness of our activity measure is likely to apply equally to the index and reference cohorts, which implies that it is a source of random error, not of bias. In some sense, our use of routine data might actually be a strength as the study in itself did not affect the clinical performance caused by the research question.

It is well known that discharge diagnoses are not entirely accurate. However, UC may have been misclassified in only approximately $10 \%$ of the cases listed in the Discharge Registry, ${ }^{18}$ and data quality for surgical procedures is high. As this is most likely evenly distributed between the cohorts, it may have led to a slight bias towards observing no difference.

Finally, an unknown proportion of our reference cohort had an appendicectomy before we had a chance to observe it-that is, before the DNRP began registering in 1977. However, this proportion is likely to be very small. The proportion of incident UC patients that had an appendicectomy prior to UC onset varies between $1 \%$ and $8 \%$ in the literature. ${ }^{1-6}$ There is no reason to suspect it should be higher in our reference cohort. In addition, we know that our reference subjects did not have an appendicectomy from 1977 to 1999. Hence the conditional probability of an unregistered appendicectomy prior to UC onset in our study is probably lower than the $1-8 \%$ known from the literature. Furthermore, this is only a problem to the extent that UC with onset many years after appendicectomy would have an atypical course.

It would be desirable to conduct a trial in which patients with UC were randomised to appendicectomy versus no appendicectomy and followed with respect to clinical outcome. Randomisation would ensure that the study groups were comparable at baseline with respect to determinants of clinical course. Unfortunately, our study does not lend support to expectations of a therapeutic breakthrough by such a trial.

With due reservation to the crudeness of our activity measure and this study being purely observational, we conclude that appendicectomy does not ameliorate the clinical course in UC patients.

\section{ACKNOWLEDGEMENTS}

We are indebted to John Baron MD for suggestions. The paper was supported by a grant from Funen County Medical Research Foundation.

\section{Authors' affiliations}

J Hallas, Department of Internal Medicine, and Department of Medical Gastroenterology, Odense University Hospital, Denmark

D Gaist, Department of Epidemiology, Institute of Public Health, University of Southern Denmark, Denmark

W Vach, Department of Statistics and Demography, University of Southern Denmark, Denmark

H T Sørensen, Department of Clinical Epidemiology, Aarhus University Hospital and Aalborg Hospital, Denmark

\section{REFERENCES}

1 Rutgeerts P, D'Haens $G$, Hiele $M$, et al. Appendectomy protects against ulcerative colitis. Gastroenterology 1994; 106:1251-3.

2 Duggan AE, Usmani I, Neal KR, et al. Appendicectomy, childhood hygiene, Helicobacter pylori status, and risk of inflammatory bowel disease: a case control study. Gut 1998;43:494-8.

3 Gent AE, Hellier MD, Grace RH, et al. Inflammatory bowel disease and domestic hygiene in infancy. Lancet 1994;343:766-7.

4 Russel MG, Dorant E, Brummer R-JM, et al. Appendectomy and the risk of developing ulcerative colitis or Crohn's disease: results of a large case-control study. Gastroenterology 1997;113:377-82.

5 Derby LE, Jick H. Appendectomy protects against ulcerative colitis. Epidemiology 1998;9:205-7.

6 Breslin NP, McDonell C, O'Morain C. Surgical and smoking history in inflammatory bowel disease: a case-control study. Inflamm Bowel Dis 1997;3:1-5.

7 Andersson RE, Olaison G, Tysk C, et al. Appendectomy and protection against ulcerative colitis. N Engl J Med 2001;344:808-14.

8 Orholm M, Munkholm P, Langholz E, et al. Familial occurrence of inflammatory bowel disease. N Engl J Med 1991;324:84-8.

9 Orholm M, Fonager K, Sørensen HT. Risk of ulcerative colitis and Crohn's disease among offspring of patients with chronic inflammatory bowel disease. Am J Gastroenterol 1999;94:3236-8.

10 Probert CSJ, Jayanthi V, Hughes AO, et al. Prevalence and family risk of ulcerative colitis and Crohn's disease: an epidemiological study among Europeans and south Asians in Leicestershire. Gut 1993;34:1547-51.

11 Andersen TF, Madsen M, Jorgensen J, et al. The Danish National Hospital Register. A valuable source of data for modern health sciences. Dan Med Bull 1999;46:263-8.

12 Greenland S, Rothman KJ. Modern epidemiology, 2nd edn. New York: Lippincott-Raven, 1998.

13 Walker AM. Observation and inference: an introduction to the methods of epidemiology. Boston: Epidemiology Resources, 1991:45-61.

14 Stata Version 7, Stata Corporation, 4905 Lakeway Drive College Station, Texas 77845 USA. http://www.stata.com.

15 Langholz E, Munkholm P, Davidsen M, et al. Course of ulcerative colitis: analysis of changes in disease activity over years. Gastroenterology 1994;107:3-11.

16 Selby WS, Griffin S, Abraham N, et al. Appendectomy protects against the development of ulcerative colitis but does not affect its course. Am J Gastroenterol 2002;97:2834-8.

17 Langholz E, Munkholm P, Nielsen $\mathrm{OH}$, et al. Incidence and prevalence of ulcerative colitis in Copenhagen county from 1962 to 1987. Scand J Gastroenterol 1991;26:1247-56.

18 Fonager K, Sorensen HT, Rasmussen SN, et al. Assessment of the diagnoses of Crohn's disease and ulcerative colitis in a Danish hospital information system. Scand J Gastroenterol 1996;31:154-9. 